\title{
Nova proposta de teste incremental de remada na avaliação aeróbia de surfistas
}

CDD. 20.ed. 796.033

797.15

http://dx.doi.org/10.1590/1807-55092015000300361
Felipe Bercht CANOZZI ${ }^{*}$ Bruno Honorato da SILVEIRA* Mariana Fernandes Mendes de OLIVEIRA* Fabrízio CAPUTO*
*Centro de Ciências da Saúde e do Esporte, Universidade do Estado de Santa Catarina.

\section{Resumo}

Os objetivos desse estudo foram: 1) verificar as respostas do lactato sanguíneo e da frequência cardíaca (FC) durante um protocolo de campo específico de remada no surfe; 2) correlacionar os índices de capacidade e potência aeróbia determinados nesse protocolo específico com o tempo de prática da modalidade e variáveis antropométricas. Participaram deste estudo nove sujeitos $(24 \pm 4,5$ anos; $72,2 \pm 6,7 \mathrm{~kg} ; 178,4 \pm 4,8 \mathrm{~cm})$ que foram submetidos a um teste progressivo intermitente de remada sobre a sua própria prancha de surfe, do tipo vai e vêm, com velocidades iniciais entre $1-1,1 \mathrm{~m} / \mathrm{s}$ e incrementos de $0,05 \mathrm{~m} / \mathrm{s}$ a cada $3 \mathrm{~min}$ até a exaustão voluntária. Uma resposta linear e exponencial foram observadas para a FC e o lactato sanguíneo, respectivamente, durante o protocolo incremental. Este comportamento foi semelhante ao demonstrado durante protocolos incrementais com objetivo de avaliar a capacidade e a potência aeróbia realizados em outras modalidades cíclicas. Além disso, foram encontradas correlações significantes entre o pico de velocidade (PV) e a velocidade correspondente ao início do acúmulo de lactato no sangue (vOBLA) $(r=0,87, p=0,005)$ e do PV com tempo de prática de surfe $(r=0,70, p=0,03)$. No entanto, não foram encontradas correlações significativas entre PV e vOBLA com nenhuma das variáveis antropométricas mensuradas. Assim, podemos concluir que o protocolo incremental específico de remada no surfe utilizado no presente estudo poderia ser uma ferramenta útil na determinação de indices relacionados à capacidade (VOBLA) e potência (PV) aeróbia de surfistas.

PalavRas-chave: Teste incremental de remada; Aptidão aeróbia; Surfe; Antropometria.

\section{Introdução}

O surfe pode ser classificado como uma modalidade esportiva intermitente, com variaçóes na intensidade e duração $o^{1-2}$. De maneira geral tem sido demonstrado que a intensidade da sessão é predominantemente moderada (64-81\% da frequência cardíaca máxima - FCmax $)^{3}$ intercalada com períodos de alta intensidade ( $>81 \%$ da $\mathrm{FCmax})^{3-6}$. A duração de uma sessão específica de surfe pode ser bastante variável, pois depende tanto da condição do mar quanto do objetivo do praticante. Em competiçóes, logo após percorrer a onda é importante que o atleta volte rapidamente para ter prioridade na escolha de uma nova onda, sendo que este processo é repetido diversas vezes. Uma bateria, momento no qual os atletas se enfrentam para definir quem tem um melhor desempenho na onda, pode durar de 20 a 40 minutos, ocorrendo mais de uma vez em um único dia de competiçãa ${ }^{1,7-8}$. Assim, é de fundamental importância um bom condicionamento aeróbio para minimizar a fadiga e maximizar a remoção de metabolitos, fatores essenciais para a manutenção do rendimento nas ondas por períodos prolongados.

Tem sido demonstrado que uma hora de surfe recreacional, apresenta um predomínio de locomoção com remada ocupando $44 \%$ do tempo total, $5 \%$ de locomoção sobre a onda, $35 \%$ sem locomoção e o restante com outras atividades como recuperar a prancha e passar por debaixo da onda ${ }^{2,5,9}$. Mendez-Villanueva et $\mathrm{al}^{2}{ }^{2}$ avaliaram 42 atletas de elite durante as baterias de uma competiçáo e demonstraram que em média foram despendidos $51,4 \%$ do tempo na locomoção de remada, $42,5 \%$ sem locomoção, $3,8 \%$ 
locomoção sobre a onda e 2,2\% outras atividades do tempo total da bateria. Apesar da diferença observada entre os estudos supracitados ambos demonstraram que a maior parte do tempo é despendido na locomoção com remada, reforçando a importância desse fundamento para a modalidade em questão.

Com relação às respostas fisiológicas, Brasil et al. ${ }^{6}$ demonstraram que a frequência cardíaca média durante uma sessão de surfe com uma hora de duraçâo foi de $135 \pm 23 \mathrm{bpm}(64 \%$ da FCmax $)$ para os surfistas do litoral paulista. Separando os diferentes estímulos durante a bateria CORRÊA et al. ${ }^{5}$ verificou que o tempo médio gasto foi de $3,2 \pm 2,2$ min parado, $1,6 \pm 1,0$ min remando e $22,9 \pm 13,1$ s de locomoção sobre a onda, com os respectivos valores de frequência cardíaca (FC) de $146 \pm 15$ bpm (77,6\% FCmax); 165 $\pm 12 \mathrm{bpm}$ (87,6\% FCmax); e $173 \pm 6 \mathrm{bpm}(92 \%$ FCmax). Apesar de não terem sido reportadas medidas que caracterizassem o metabolismo anaeróbio durante o surfe recreacional ou de competição, os breves períodos de locomoçáo sobre a onda são de alta intensidade, o que exige uma participação das reservas anaeróbias principalmente na parte inicial (remada para entrada na onda) e durante os movimentos de elevada potência durante as manobras. Dessa forma, podemos caracterizar o surfe como uma modalidade de intensidade moderada intercalada com períodos de exercícios de alta intensidade e potência demandando tanto o metabolismo aeróbio quanto o anaeróbio ${ }^{2}$.

Os estudos citados anteriormente demonstram uma predominância do metabolismo aeróbio durante a prática do surfe. Sendo assim, a demanda fisiológica do organismo deve ser analisada não apenas na execução das manobras, mas durante toda bateria $^{2,9}$, visto que os surfistas ocupam a menor parte do tempo com movimentos sobre a onda, ou seja, em alta intensidade. Reforçando a importância do desenvolvimento da capacidade aeróbia para surfistas, Mendez-Villanueva et al. ${ }^{8}$ encontraram uma correlação significativa $(\mathrm{r}=0,58, \mathrm{p}=0,03)$ entre o início do acúmulo de lactato no sangue OBLA (intensidade correspondente a concentraçáo fixa de $4 \mathrm{mM}$ de lactato sanguíneo) e a classificação

\section{Método}

\section{Delineamento experimental}

Os sujeitos foram orientados a se apresentarem nos testes descansados, alimentados, hidratados e não final obtida durante uma temporada competitiva, sugerindo que o desempenho do surfista está relacionado a sua capacidade aeróbia ${ }^{1,9-10}$.

Apesar de existir controvérsias em relação aos diferentes métodos usados para interpretar as modificaçóes nos níveis de lactato durante o exercício, o OBLA tem demonstrado ser um bom indicador de desempenho em provas aeróbias de diferentes duraçóes como também nas modalidades intermitentes $^{1,11-12}$. No entanto, poucos estudos a respeito destes indicadores fisiológicos foram feitos em atividades que usam predominantemente os membros superiores e de natureza intermitente como a remada no surfe ${ }^{8,10,13}$. Recentemente, Mendez-Villannueva et al. ${ }^{8}$ determinaram o OBLA $(4 \mathrm{mM})$ durante um teste incremental de simulação de remada sobre a prancha em um grupo de 13 surfistas competitivos. Os autores encontram valores significantemente maiores de OBLA para surfistas de alto nível em relação aos surfistas de nível regional.

$\mathrm{Na}$ tentativa de aproximar a especificidade da remada, surfistas têm sido avaliados em ergômetro de braço e ergômetro específico para remada ${ }^{2,9}$. No entanto, poderia ainda existir diferenças no gesto motor realizado no ergômetro de laboratório com a prática de campo, além da limitaçáo do teste fora do ambiente aquático. $\mathrm{O}$ equilíbrio, o controle da direção de remada, e o tônus das musculaturas acessórias podem diferenciar de maneira específica às habilidades envolvidas numa sessão de surfe ${ }^{1,2,8}$. Até o presente momento náo foram encontrados estudos que utilizaram um protocolo específico de campo para avaliação da aptidão aeróbia de surfistas, visto que tanto a capacidade quanto à potência aeróbia parecem influenciar no desempenho do surfe ${ }^{1-2,9}$. Assim, os objetivos desse estudo foram: 1) verificar o comportamento das variáveis fisiológicas lactato sanguíneo e FC durante um protocolo de campo específico de remada no surfe desenvolvido para avaliar a aptidáo aeróbia de surfistas; 2) correlacionar os índices de capacidade (vOBLA) e potência aeróbia (pico de velocidade - PV) determinados nesse protocolo específico com o tempo de prática da modalidade e variáveis antropométricas.

realizar esforços intensos nas 24 horas antecedentes. Todos os procedimentos foram realizados em uma única visita, seguindo a ordem: 1) apresentação dos procedimentos experimentais e suas implicações, 
registro do tempo de prática (anos) na modalidade e assinatura do termo de consentimento para a participação no estudo; 2) avaliação antropométrica e; 3) teste incremental de remada. O teste de remada foi realizado em piscina aquecida e coberta com a temperatura da água mantida em $29 \pm 1{ }^{\circ} \mathrm{C}$. Essa pesquisa foi autorizada pelo Comitê de Ética em Pesquisas com Seres Humanos da Universidade do Estado de Santa Catarina sob o n. 167/2009.

\section{Sujeitos}

Participaram do estudo nove surfistas recreacionais, com um tempo de prática mínimo de três anos e que realizavam pelo menos uma sessão semanal. As características antropométricas e descrição do treinamento de cada sujeito se encontram descritos na TABELA 1.

\section{Teste aeróbio de remada}

Os sujeitos foram submetidos a um teste progressivo intermitente de remada na sua própria prancha de surfe ("Shortboard" ou "Pranchinha" com tamanho recomendado para surfar ondas de 1 a 1,5 m), do tipo vai e vem, com velocidade inicial podendo variar de $1 \mathrm{~m} / \mathrm{s}, 1,05 \mathrm{~m} / \mathrm{s}$ ou $1,1 \mathrm{~m} / \mathrm{s}$ conforme o nível de condicionamento físico do indivíduo, e incrementos de $0,05 \mathrm{~m} / \mathrm{s}$ a cada estágio até a exaustão voluntária. A intensidade do teste foi controlada por sinais sonoros emitidos a cada $10 \mathrm{~s}$, assim o sujeito deveria percorrer a distância estipulada (e.g. $10 \mathrm{~m}$ para o estágio de $\mathrm{m} / \mathrm{s}$ ) iniciando e terminando em sincronia com os sinais sonoros. Ao final de cada percurso houve um período de $5 \mathrm{~s}$ de recuperação no qual foi solicitado ao voluntário que girasse sobre seu eixo $\left(180^{\circ}\right)$ posicionando seu corpo de frente para realização de um novo percurso permanecendo sentado até que o novo sinal sonoro fosse emitido. Esse procedimento foi repetido 12 vezes para cada estágio totalizando um período de três minutos. $\mathrm{O}$ teste foi interrompido por exaustão voluntária ou quando o sujeito atrasasse em $1 \mathrm{~m}$ o término do percurso por duas vezes consecutivas. Ao final de cada estágio houve um intervalo de 30 segundos no qual foram coletadas amostras de sangue do lóbulo de orelha para análise da concentração de lactato. A velocidade associada ao início do acúmulo de lactato no sangue (vOBLA) associada a concentração fixa de $3,5 \mathrm{mM} / \mathrm{L}$ foi determinada por interpolação linear entre as velocidades e suas respectivas concentraçóes de lactato. A frequência cardíaca foi registrada em intervalos de cinco segundos durante todo teste (Polar S610i, Polar, Kempele, Finlândia). Para controle do teste, foi utilizado um amplificador de som e um "notebook" com o programa desenvolvido para gerar sinais sonoros sistematizados Windows Movie Maker, com precisão de 0,04 segundos.

Foi considerada como frequência cardíaca máxima $\left(\mathrm{FC}_{\max }\right)$ o maior valor de $\mathrm{FC}$ registrada durante o teste. A maior velocidade atingida foi considerada como pico de velocidade (PV). Caso sujeito interrompesse o teste antes de finalizar o estágio, o PV $\left(\mathrm{m} . \mathrm{s}^{-1}\right)$ foi corrigido a partir da seguinte equação:

$\mathrm{PV}=\mathrm{v}+[(t i / t) \bullet 0,05]$

Onde: v é a velocidade do último estágio completo em $\mathrm{m} . \mathrm{s}^{-1}$, o ti é tempo realizado no estágio incompleto, $t$ é tempo total do estágio e $0,05 \mathrm{~m} \cdot \mathrm{s}^{-1}$ o incremento da velocidade no estágio adaptado de Kuipers et al. ${ }^{14}$.

\section{Avaliação antropométrica}

Antes do teste progressivo os indivíduos foram submetidos à avaliação antropométrica na qual a massa corporal foi medida por meio de uma balança digital marca Toledo ${ }^{\oplus}$ com precisão de 50 gramas, a estatura por meio de um estadiômetro profissional Sanny ${ }^{\circledR}$ e a envergadura por meio de fita métrica Western ${ }^{\circledast}$. As dobras cutâneas (tricipital, peitoral, abdominal e subescapular), foram avaliadas através de um adipômetro científico da marca Cescorf ${ }^{\oplus}$ com precisão de $0,1 \mathrm{~mm}$. Para o cálculo da estimativa do percentual de gordura corporal (\%G), a equação de $\operatorname{LOHMAN}^{15}$.

\section{Análise estatística}

Todas as variáveis analisadas no estudo estão apresentadas como média \pm desvio padrão (DP). Os dados apresentaram distribuição normal verificada através do teste de Shapiro-Wilk. A correlação entre os índices fisiológicos e as variáveis antropométricas foi determinada através do coeficiente de correlação de Pearson. Em todos os testes foi adotado um nível de significância de $\mathrm{p}<0,05$. 


\section{Resultados}

Na TABELA 1 estão representados valores individuais, média e desvio padrão (DP) das características antropométricas (idade, estatura, massa corporal, envergadura, somatória das dobras cutâneas - $\sum \mathrm{e}$ $\% \mathrm{G}$ ) e caracterização do treinamento (tempo total de treinamento e volume semanal de treino) de nove surfistas recreacionais da cidade de Florianópolis.

Os valores individuais das variáveis fisiológicas bem como sua relaçáo com as cargas (velocidade) obtidas durante o teste de remada estão descritos na TABELA 2.

TABELA 1- Valores individuais, média \pm DP das características dos sujeitos antropométricas e do treinamento.

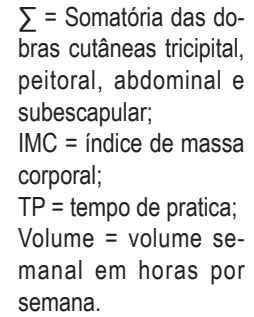

$\sum$ Somatória das dopeitoral, abdominal e subescapular;

MC = indice de massa $\mathrm{TP}=$ tempo de pratica Volume = volume sesemana.

\begin{tabular}{lcccccccc}
\hline Sujeitos & $\begin{array}{c}\text { Idade } \\
(\mathbf{a n o s})\end{array}$ & $\begin{array}{c}\text { Estatura } \\
(\mathbf{c m})\end{array}$ & $\begin{array}{c}\text { Massa } \\
(\mathbf{k g})\end{array}$ & $\begin{array}{c}\text { Envergadura } \\
(\mathbf{c m})\end{array}$ & $\begin{array}{c}\Sigma \\
(\mathbf{m m})\end{array}$ & $\begin{array}{c}\text { IMC } \\
\left(\mathbf{k g} / \mathbf{m}^{\mathbf{2}}\right)\end{array}$ & $\begin{array}{c}\text { TP } \\
(\mathbf{a n o s})\end{array}$ & $\begin{array}{c}\text { Volume } \\
(\mathbf{h} / \mathbf{s e m})\end{array}$ \\
\hline 1 & 21 & 180,0 & 79,3 & 184 & 31,6 & 24,3 & 6 & 16 \\
2 & 35 & 181,0 & 77,4 & 186 & 25,9 & 23,6 & 25 & 10 \\
3 & 28 & 176,6 & 65,1 & 167,7 & 18,2 & 20,9 & 15 & 8 \\
4 & 21 & 168,7 & 65,1 & 185 & 34,9 & 22,9 & 8 & 10 \\
5 & 22 & 180,6 & 70,3 & 175,9 & 14,5 & 21,6 & 7 & 1,5 \\
6 & 22 & 181,0 & 69,2 & 186,3 & 16,3 & 21,1 & 8 & 4,5 \\
7 & 21 & 177,4 & 72,5 & 187 & 23,4 & 23,0 & 3 & 4,5 \\
8 & 23 & 174,4 & 66,8 & 182 & 22,7 & 22,0 & 12 & 4,5 \\
9 & 22 & 185,3 & 84,0 & 186,2 & 25,3 & 24,5 & 10 & 6 \\
\hline Média & 24,2 & 178,4 & 72,2 & 182,2 & 23,6 & 22,6 & 10,4 & 7,3 \\
DP & 4,5 & 4,8 & 6,7 & 6,4 & 6,7 & 1,3 & 6,4 & 4,3 \\
\hline
\end{tabular}

TABELA 2 - Valores individuais, média \pm DP das variáveis fisiológicas obtidas durante o teste incremental de remada no surfe.

$\mathrm{PV}=$ pico de velocidade; $\mathrm{FC}_{\text {max }}=$ frequência cardíaca máxima;

[La]pico = pico da concentração de lactato;

VOBLA = velocidade referentes ao inicio de acumulo de lactato no sangue expresso em m.s-1;

$\% \mathrm{OBLA}=$ valores percentuais da vOBLA relativo ao PV;

FC e OBLA, frequência cardíaca na $\mathrm{VOBLA}$ expressa de forma absoluta (bpm) e relativa a $\mathrm{FCmax}\left(\% \mathrm{FC}_{\max }\right)$.

8

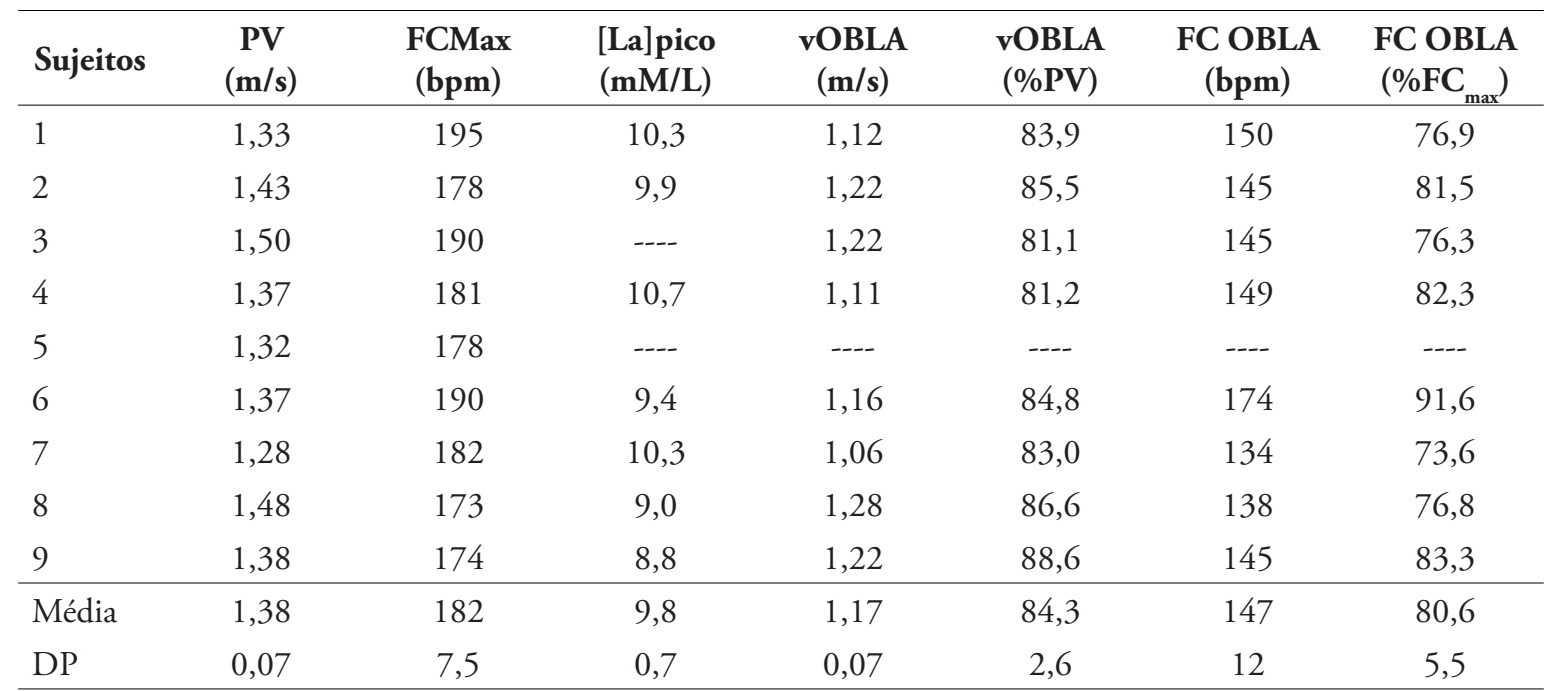

O comportamento da FC e do lactato sanguíneo durante o teste incremental de remada de um sujeito representativo (painéis superiores) e da média do grupo (painéis inferiores) com os respectivos desvios padrão estão representados na FIGURA 1.
Foram encontradas correlaçôes significativas entre o PV e a vOBLA $(r=0,87, p=0,005)$ (FIGURA 2) e do tempo de prática (TP) na modalidade com o PV $(\mathrm{r}=0,70, \mathrm{p}=0,03)$ (FIGURA 3). Porém, não foram encontradas correlaçóes significativas entre PV e vOBLA com nenhuma das variáveis antropométricas mensuradas, nem com o volume semanal de treino. 

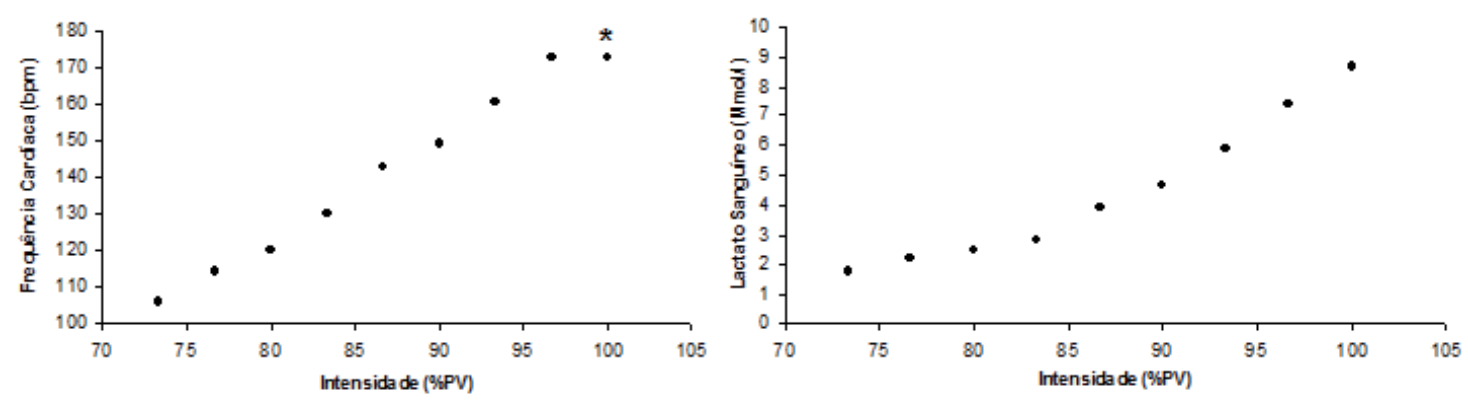

* o sujeito permaneceu 117 s no último estágio.
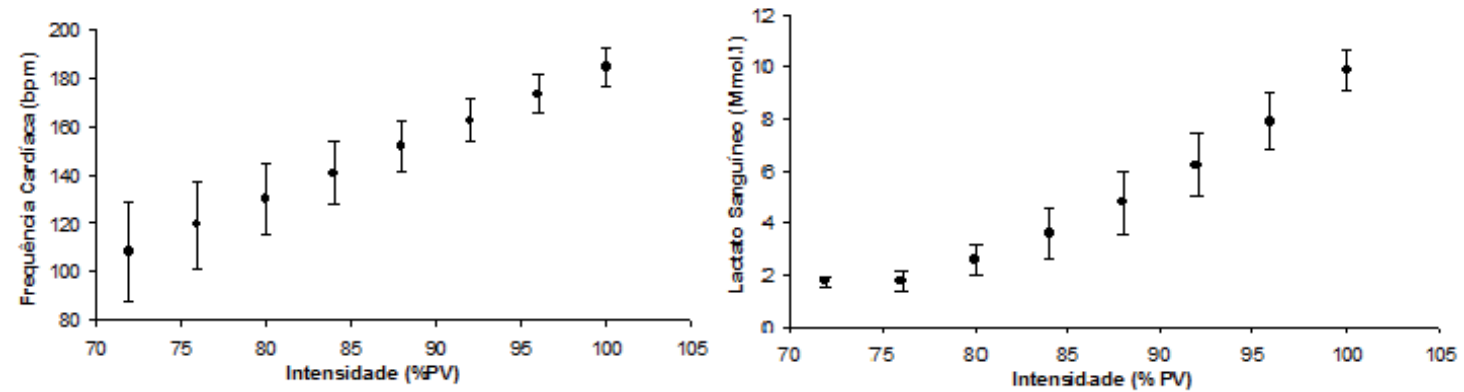

FIGURA 1 - Respostas da frequência cardíaca (FC) elactato sanguíneo durante o testeincremental de remada para um sujeito representativo (painéis superiores) e a para a média \pm DP do grupo (painéis inferiores).

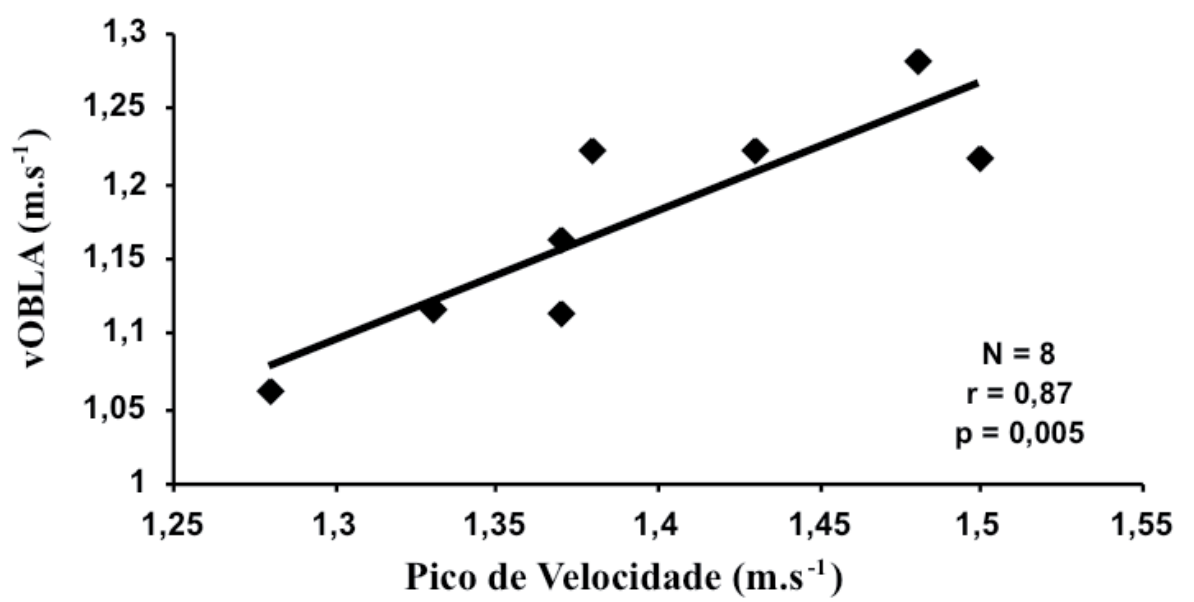

FIGURA 2 - Correlação entre a velocidade no início do acúmulo de lactato no sangue (vOBLA) e o Pico de Velocidade (PV) obtidos durante teste incremental.

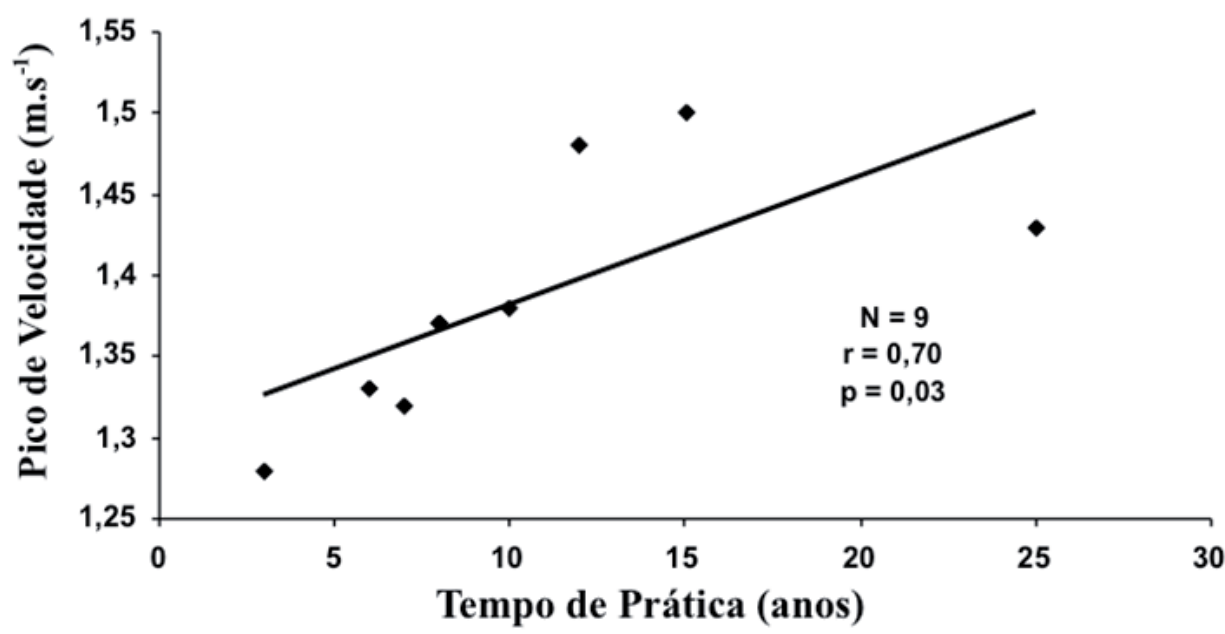

FIGURA 3 - Correlação entre o Pico de Velocidade (PV) e o tempo de prática na modalidade. 


\section{Discussão}

Foi demonstrado no presente estudo que as respostas da FC e do lactato sanguíneo apresentaram um comportamento linear e exponencial, respectivamente, semelhante ao demonstrado durante protocolos incrementais realizados em outras modalidades cíclicas com objetivo de avaliar a capacidade e a potência aeróbia $^{8,13,16-17}$. Foi também verificada uma elevada correlaçáo entre o PV e a vOBLA. Esses resultados em conjunto indicam que o protocolo específico de campo desenvolvido no presente estudo poderia ser usado para avaliação da aptidão aeróbia de surfistas. Além disso, a ausência de correlação entre as variáveis fisiológicas (PV e vOBLA) e o volume de treino semanal sugere que as adaptaçóes fisiológicas específicas parecem sofrer maior influência da intensidade do treinamento que do volume de treinamento. Assim, a intensidade da sessão seria a variável mais influente no rendimento da remada no surfe e no PV.

Em relação à estatura e massa corporal, Mendez-Villanueva et al. ${ }^{8}$ relatou que surfistas competidores demonstram valores menores quando comparados a populaçóes de outros esportes de mesma faixa etária. A média de estatura para os surfistas "top" 34 do "ranking" de surfistas profissionais da ASP (Association of Surfing Professionals) $)^{18}$ participantes do WCT 2011 (World Competition Tour - 1a divisão do "surf" profissional) foi $177,4 \pm 5,8 \mathrm{~cm}$. Estes valores são semelhantes aos dados do presente estudo $(178,4 \pm 4,8 \mathrm{~cm})$ e ligeiramente superiores ao estudo de LowDON ${ }^{19}$ que relatou média de estatura de $173,6 \pm 5,9 \mathrm{~cm}$ para 76 surfistas internacionais. Em conjunto os dados acima apresentados demonstram que os surfistas parecem ter uma menor estatura que os nadadores de elite $(183,8 \pm 7,1 \mathrm{~cm} ; \mathrm{n}=231)$ e os jogadores de pólo aquático $(186,5 \pm 6,5 \mathrm{~cm} ; \mathrm{n}$ $=190)^{20}$. A menor estatura verificada para surfistas pode estar relacionada com o fato de a estabilidade ser inversamente proporcional a elevaçáo do centro de massa. Dessa forma, uma menor estatura, representa uma vantagem para o rendimento do surfista, um centro de gravidade mais baixo favorece ao equilíbrio dinâmico, sendo um fator essencial na retomada de equilíbrio durante as manobras 5 .

Em relação à massa corporal, LowDON ${ }^{19}$ avaliou 76 surfistas de nível internacional e encontrou um valor de $67,9 \mathrm{~kg}$. O presente estudo demonstrou valores superiores $(73,3 \mathrm{~kg})$, porém semelhantes aos dados dos surfistas "top" 34 do "ranking" de surfistas profissionais da ASP (Association of Surfing Professionals) participantes do WCT 2011 (World
Competition Tour - $1^{\text {a }}$ divisão do "surf" profissional) que foi de $74 \pm 5,3 \mathrm{~kg}$. Apesar de haver uma pequena diferença entre os surfistas avaliados por LowDON ${ }^{19}$ e os dados mais atuais, maiores diferenças são verificadas quando comparamos os surfistas aos atletas de outras modalidades aquáticas relatadas por MAzZA et al. ${ }^{20}$, com valores de massa de $78,4 \mathrm{~kg}$ e $86,1 \mathrm{~kg}$ para nadadores $(\mathrm{n}=231)$ e atletas de pólo aquático $(\mathrm{n}=$ 190), respectivamente. Assim, os valores de massa corporal relativamente menor em surfistas, podem estar relacionados a uma menor estatura além de ser uma vantagem no desenvolvimento das açóes específicas do surfe, já que com uma maior massa há necessidade de maior força para realizar a aceleraçãoo ${ }^{1,8}$.

A análise da composição corporal de surfistas tem demonstrado uma grande variação de gordura corporal entre os sujeitos, sugerindo não haver relaçáo relevante desta variável no rendimento dos surfistas ${ }^{19}$. Geralmente, os valores de percentual de gordura corporal de surfistas são mais altos que atletas de alto rendimento em modalidades aeróbias, porém mais baixos que a média de idade de indivíduos não atletas. Lowdon e PATEMAN ${ }^{21}$ relataram médias de $10,5 \%$ para 16 surfistas de nível internacional masculinos, semelhante a média de $10,4 \%$ para surfistas recreacionais encontrada em nosso estudo. Segundo LowDON ${ }^{22}$ como a massa do surfistas é suportada sobre a prancha durante a remada e o percorrer da onda, uma baixa gordura corporal não representaria uma real vantagem sobre o rendimento, como poderia ocorrer durante a corrida ou ciclismo em subida. Nossos dados corroboram com este raciocínio, uma vez que não foi encontrada correlação significativa entre as variáveis antropométricas (\%G e $\Sigma$ ) e as variáveis fisiológicas estudadas, sugerindo que durante a remada, mesmo com períodos frequentes de aceleraçáo, estes aspectos náo interferiram no rendimento da remada na amostra e condiçẫo estudada. No entanto, ainda seriam necessários mais estudos para observar se essa relação também ocorre em surfistas profissionais.

Alguns estudos têm indicado que a utilização de uma concentração fixa de lactato $(3,5$ ou $4 \mathrm{mM})$ para estimar a máxima fase estável de lactato sanguíneo (MFEL) pode náo ser apropriada, pois o estado nutricional ${ }^{23}$, a variabilidade individual ${ }^{16}$ e o tipo de exercício ${ }^{24}$, podem influenciar nesta predição. Entretanto, DenadaI et al. ${ }^{11}$ verificaram em cicloergômetro que a validade do OBLA para estimar a intensidade de MFEL é independente do estado de treinamento aeróbio (ativos $\mathrm{x}$ treinados). Além 
disso, diversos estudos têm mostrado que o OBLA é valido para a predição do desempenho aeróbio na natação ${ }^{25-26}$, embora poucos estudos compararam o OBLA com a MFEL particularmente em ambiente aquático $^{27}$. Desta forma, podemos sugerir que o OBLA utilizado no presente estudo, independente da sua validade em estimar MFEL, pode ser um índice que reflete a capacidade aeróbia do sujeito.

O PV tem se mostrado um ótimo preditor de desempenho aeróbio e também sensível aos efeitos do treinamento em atletas, mesmo quando o $\mathrm{VO}_{\text {2pico }}$ não se altera, pois pode haver melhora da economia de movimento ${ }^{28}$. Semelhante a resposta do lactato ao exercício incremental (OBLA), que por depender mais das adaptaçóes periféricas (capacidade oxidativa muscular), apresenta uma grande sensibilidade aos efeitos do treinamento e também uma alta correlação com o desempenho aeróbio em eventos de média e longa duração ${ }^{29}$. A elevada correlação encontrada entre o PV e a vOBLA demonstra que o protocolo utilizado em nosso estudo foi capaz de discriminar diferenças na aptidão aeróbia entre os indivíduos, quando observados estes indicadores de potência (PV) e capacidade (OBLA) aeróbia. Resultados semelhantes foram também encontrados por KRUSTRUP et al. ${ }^{17}$ que observaram uma correlação inversa entre a concentração submáxima de lactato e a performance durante um teste incremental intermitente (Yo-Yo Intermittent Recovery Test). Esses dados confirmam a importância da determinação do PV principalmente em situaçóes na qual o $\mathrm{VO}_{2}$ não pode ser mensurado, reforçando os achados de outros estudos, nos quais a velocidade associada ao $\mathrm{VO}_{\text {2pico }}\left(\mathrm{IVO}_{\text {2pico }}\right)$ explicou melhor as diferenças individuais na performance aeróbia do que o $\mathrm{VO}_{2 \text { pico }}$ ou a economia de movimento isoladamente ${ }^{30}$. Como no presente estudo não avaliamos performance de remada na condiçáo real, bem como performance no surfe, outros estudos ainda são necessários para confirmar a validade destes índices (PV e OBLA) obtidos em um protocolo específico de campo para predição de performance e detecção dos efeitos do treinamento.

O rendimento no surfe é bastante influenciado pelas diferentes condições externas, como tipo, tamanho e comprimento da onda, correnteza, entre outros fatores. Devido à natureza imprevisível dos fatores externos desta modalidade, torna-se difícil o controle da intensidade de trabalho durante o período de remada, e posterior quantificação do volume e intensidade de treino e suas relaçóes com o rendimento. Como regra geral os surfistas retornam para área de competição o mais rápido possível.
Desta forma, durante a prática do surfe é provável que os surfistas que se deparem com condições externas mais difíceis responderão melhor a uma determinada situação, apesar de não haver diferenças no volume entre os surfistas amadores e profissionais quando levado em consideração à prática semanal ${ }^{1{ }^{1,8}}$. Alguns estudos têm demonstrado que as melhoras tanto na PV e OBLA são mais dependentes da intensidade do que o volume de treinamento, requerendo intensidades bem elevadas para ocasionar melhoras nestes índices ${ }^{12,31-32}$. Corroborando com esses achados, Mendez-Villanueva et al. ${ }^{8}$ encontraram maiores valores de potência pico (PP) e OBLA determinados em um ergômetro de remo adaptado para surfistas de alto nível comparado aos de nível regional, apesar da semelhança no $\mathrm{VO}_{2 \max } \mathrm{e}$ frequência de treino semanal. Além disso, os autores observaram correlação significante de ambos PP e OBLA com a classificação final em uma temporada ${ }^{8}$. No presente estudo foi encontrada uma elevada correlação entre PV e OBLA, demonstrando que os indivíduos com os maiores valores de PV também possuíam valores elevados de OBLA. Além disso, foi também encontrada correlação positiva entre os anos de prática na modalidade e o PV. Assim, surfistas com maior rendimento e mais experiência provavelmente são submetidos mais frequentemente a altas intensidades durante a sua prática esportiva e, portanto, alcançam maiores adaptaçóes.

É possível que um maior valor de vOBLA possa atrasar os prejuízos nas atividades motoras finas induzidas pela fadiga. A fadiga induzida pelo exercício de membros superiores pode ter uma influência sobre o rendimento do surfe. Em seu estudo, BogDanis et al. ${ }^{33}$ observaram o rendimento de um exercício de pernas (repetidos esforços de alta intensidade no ciclismo) precedido ou não por um exercício de braços e encontraram uma significante reduçáo na potência pico e potência média quando precedido pelo exercício de braços. Portanto, é possível que a fadiga induzida durante a remada possa estar associada com alguns efeitos negativos sobre o controle postural e desempenho ao surfar uma onda, em pé sobre a prancha e realizando manobras com a maior velocidade e maior grau de dificuldade. Como a maioria dos períodos de remada e de surfe não é de escolha do atleta, uma boa preparação para sustentar remadas intermitentes com períodos de alta intensidade com menor fadiga, poderia reduzir os efeitos negativos no desempenho das manobras durante uma onda surfada subsequentemente, dessa forma seria um importante aspecto na performance do surfe. 
Com base nos nossos resultados podemos concluir que o teste incremental de remada para o surfe proposto no presente estudo, apesar da sua característica intermitente, apresentou um comportamento de FC e lactato sanguíneo semelhante a protocolos incrementais utilizados em outras modalidades como natação, corrida e ciclismo. Além disso, o protocolo foi capaz de discriminar os níveis de aptidão aeróbia entre os surfistas recreacionais, uma vez que os sujeitos com os maiores valores de vOBLA foram aqueles com maiores valores de PV e os sujeitos com mais tempo de prática também apresentaram maiores PV. Desta forma, surfistas com melhores rendimentos (PV e OBLA) e maior experiência (anos de prática) provavelmente são submetidos mais frequentemente a altas intensidades durante a sua prática esportiva e assim atingem maiores adaptaçóes fisiológicas.

\begin{abstract}
A new proposed incremental surfing paddle test to assess aerobic fitness in surfers

The aim of this study were: 1) to analyse heart rate (HR) and blood lactate (La) responses during a protocol of intermittent surfing paddle on board; 2) to correlate the aerobic capacity and power indexes determined from this specific protocol with the experience in surfing practice and anthropometric variables. Participated in the study nine subjects $(24 \pm 4.5$ years; $72.2 \pm 6.7 \mathrm{~kg} ; 178.4 \pm 4.8 \mathrm{~cm})$ were submitted to an intermittent incremental shuttle paddle test on the surfboard, with initial velocity between 1 to $1.1 \mathrm{~m} / \mathrm{s}$ and increments from $0.05 \mathrm{~m} / \mathrm{s}$ for each $3 \mathrm{~min}$ until volitional exhaustion. During the incremental protocol, linear and exponential responses were observed for HR and La, respectively. These responses were similar to those demonstrated during incremental protocols in cyclic exercise modes aiming to assess the aerobic power and capacity. They were found significant correlations between the peak velocity (PV) and velocity at the Onset Blood Lactate Accumulation (vOBLA) ( $r=0.87)$ and between the PV and experience in surfing practice $(r=0.7)$. However, they were found no significant correlations between PV and VOBLA with anthropometrics variables and weekly training volume. Thus, we conclude that the specific protocol of intermittent surfing paddle could be a useful tool to determine indices related with aerobic power (PV) and capacity (OBLA) of surfers.
\end{abstract}

KeY WoRDS: Incremental surfing paddle; Aerobic fitness; Surfing; Anthropometric.

\title{
Referências
}

1. Mendez-Villanueva A, Bishop D. Physiological aspects of surfboard riding performance. Sports Med. 2005;35:55-70.

2. Mendez-Villanueva A, Bishop D, Hamer P. Activity profile of world-class professional surfers during competition: a case study. J Strength Cond Res. 2006;20:477-82.

3. Heyward, VH. Advanced fitness, assessment and exercise prescription. 3rd ed. Champaign: Human Kinetics; 1997.

4. Silva MBO. Estudo exploratório dos aspectos desportivos e médicos do surfe. Rev Bras Med Esporte. 1984;2:25.

5. Corrêa FMF, Figueira Júnior AJ, Ferreira M, Matsudo VKR. Perfil de aptidão física de surfistas profissionais brasileiros. XV Encontro Mineiro de Atividade Física; 2013; Poços de Caldas, BR. Poços de Caldas; 1993. p.18.

6. Brasil FK, Andrade DR, Oliveira LC, Ribeiro MA, Matsudo VKR. Freqüência cardíaca e tempo de movimento durante o surfe recreacional: estudo piloto. R Bras Ci Mov. 2001;9:65-75.

7. Frank M, Zhouemail S, Bezerra P, Crowley Z. Effects of long-term recreational surfing on control of force and posture in older surfers: a preliminary investigation. J Exerc Sci Fit. 2009;7:31-8.

8. Mendez-Villanueva A, Perez-Landaluce J, Bishop D, et al. Upper body aerobic fitness comparison between two groups of competitive surfboard riders. J Sci Med Sport. 2005;8:43-51.

9. Meier RA, Lowdon BJ, Davie AJ. Hearts rates and estimated energy expenditure during recreational surfing. Aust J Sci Med Sport. 1991;23:70-4. 
10. Lowdon BJ, Bedi JF, Horvath SM. Specificity of aerobic fitness testing of surfers. Aust J Sports Med. 1989;21:7-10.

11. Denadai BS, Figueira TR, Favaro OR, Goncalves M. Effect of the aerobic capacity on the validity of the anaerobic threshold for determination of the maximal lactate steady state in cycling. Braz J Med Biol Res. 2004;37:1551-6.

12. Denadai BS, Ortiz MJ, Greco CC, de Mello MT. Interval training at $95 \%$ and $100 \%$ of the velocity at VO2 max: effects on aerobic physiological indexes and running performance. Appl Physiol Nutr Metab. 2006;31:737-43.

13. Rechichi C, Dawson B, Lawrence SR. A multistage shuttle swim test to assess aerobic fitness in competitive water polo players. J Sci Med Sport. 2000;3:55-64.

14. Kuipers H, Verstappen FT, Keizer HA, Geurten P, van Kranenburg G. Variability of aerobic performance in the laboratory and its physiologic correlates. Int J Sports Med. 1985;6:197-201.

15. Lohman TG. Skinfolds and body density and their relation to body fatness: a review. Human Biology: The International Journal of Population Biology and Genetics. 1981; 53: 181-225.

16. Stegmann H, Kindermann W, Schnabel A. Lactate kinetics and individual anaerobic threshold. Int J Sports Med. 1981;2:160-5.

17. Krustrup P, Mohr M, Amstrup T, et al. The yo-yo intermittent recovery test: physiological response, reliability, and validity. Med Sci Sports Exerc. 2003;35:697-705.

18. Association of Surfing Professionals [homepage]. Los Angeles: WSL. [cited 2011 Mar 12]. Available from: http:// www.worldsurfleague.com/2010/.

19. Lowdon BJ. The somatotype the international surfboards riders. Aust J Sports Med. 1980;12: 34-9.

20. Mazza JC, Ackland TR, Bach TM. Absolute body size. In: Carter JEL, Ackland TR, editors. Kinanthropometry in aquatic sports: a study of world-class athletes. Champaign: Human Kinetics; 1994. p.15-54. (HK Sport Science Monograph Series 5).

21. Lowdon BJ, Pateman NA. Physiological parameters of international surfers. Aust J Sports Med. 1980;12:30-3.

22. Lowdon BJ. Fitness requirements for surfing. Sports Coach. 1983;6:35-8.

23. Maassen N, Busse MW. The relationship between lactic acid and work load: a measure for endurance capacity or an indicator of carbohydrate deficiency? Eur J Appl Physiol Occup Physiol. 1989;58:728-37.

24. Beneke R, Von Duvillard SP. Determination of maximal lactate steady state response in selected sports events. Med Sci Sports Exerc. 1996;28:241-6.

25. Wakayoshi K, Yoshida T, Udo M, et al. A simple method for determining critical speed as swimming fatigue threshold in competitive swimming. Int J Sports Med. 1992;13:367-71.

26. Wakayoshi K, Yoshida T, Udo M, et al. Does critical swimming velocity represent exercise intensity at maximal lactate steady state? Eur J Appl Physiol Occup Physiol. 1993;66:90-5.

27. Pereira RR, Zagatto AM, Papoti M, Gobatto CA. Validação de dois protocolos para determinação do limiar anaeróbio em natação. Motriz. 2002;8:63-8.

28. Billat VL, Mille-Hamard L, Demarle A, Koralsztein JP. Effect of training in humans on off- and on-transient oxygen uptake kinetics after severe exhausting intensity runs. Eur J Appl Physiol. 2002;87:496-505.

29. Coyle EF. Integration of the physiological factors determining endurance performance ability. Exerc Sport Sci Rev. 1995;23:25-63.

30. Daniels J, Daniels N. Running economy of elite male and elite female runners. Med Sci Sports Exerc. 1992;24:483-9.

31. Londeree BR. Effect of training on lactate/ventilatory thresholds: a meta-analysis. Med Sci Sports Exerc. 1997;29:837-43.

32. Lindsay FH, Hawley JA, Myburgh KH, Schomer HH, Noakes TD, Dennis SC. Improved athletic performance in highly trained cyclists after interval training. Med Sci Sports Exerc. 1996;28:1427-34.

33. Bogdanis GC, Nevill ME, Lakomy HK. Effects of previous dynamic arm exercise on power output during repeated maximal sprint cycling. J Sports Sci. 1994;12:363-70.

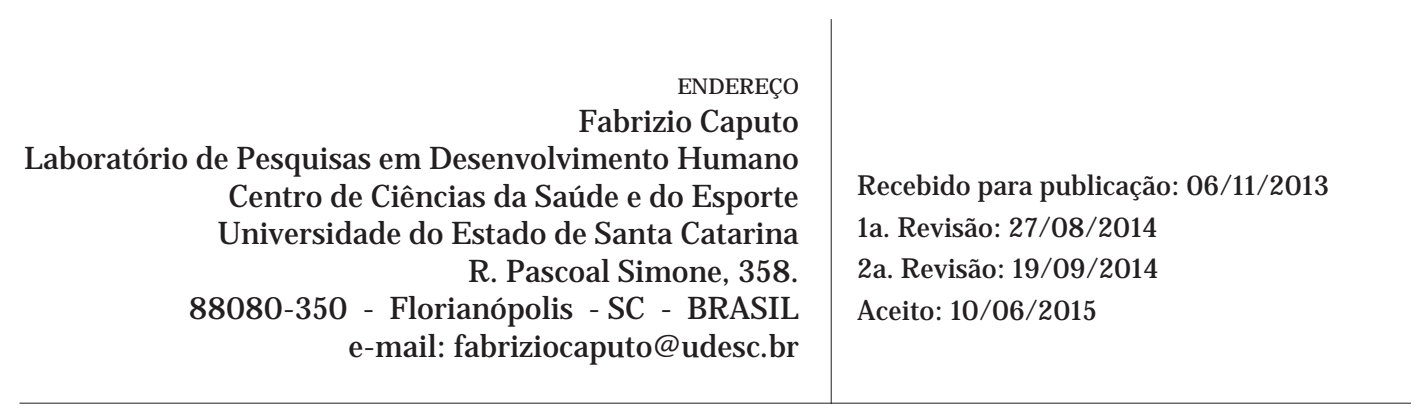

